\title{
ST-Elevation Myocardial Infarction Presenting as Acute Limb Ischemia
}

\author{
Tamoor Ahmed ${ }^{1}$, Talha Ahmed ${ }^{2}$, Reyaz Haque ${ }^{3}$ \\ 1. Internal Medicine, King Edward Medical University/Mayo Hospital, Lahore, PAK 2. Internal Medicine, University of \\ Maryland Medical Center, Baltimore, USA 3. Internal Medicine/Cardiovascular Medicine/Interventional Cardiology, \\ University of Maryland School of Medicine, Baltimore, USA
}

Corresponding author: Talha Ahmed, atalha858@yahoo.com

\begin{abstract}
We describe a case of delayed presentation of ST-segment elevation myocardial infarction (STEMI) complicated by ventricular thrombus and peripheral embolization causing limb ischemia. Our patient initially presented with symptoms of acute limb ischemia. However, on preoperative evaluation, STEMI was diagnosed. He required emergent revascularization of the left anterior descending artery followed by thrombectomy of the femoral artery. The cause of the limb ischemia was deemed to be a late presenting STEMI that was complicated by left ventricular thrombus, hence causing lower extremity embolization. Delayed presentations and complications related to STEMI may manifest as acute limb ischemia in the setting of ventricular thrombus formation and subsequent distal embolization.
\end{abstract}

Received 09/01/2020

Review began 09/07/2020 Review ended 09/11/2020 Published 09/13/2020

๑) Copyright 2020

Ahmed et al. This is an open access article distributed under the terms of the Creative Commons Attribution License CC-BY 4.0., which permits unrestricted use, distribution, and reproduction in any medium, provided the original author and source are credited.
Categories: Cardiology, Internal Medicine

Keywords: st-elevation myocardial infarction (stemi), ventricular thrombus, acute limb ischemia

\section{Introduction}

Left ventricle (LV) thrombus is not an uncommon complication of acute myocardial infarction (MI) and is often associated with systemic thromboembolism. Important risk factors include elderly patients with large anterior MI and those with delayed reperfusion [1]. The incidence may be as high as $15 \%$ in patients with STsegment elevation MI (STEMI) and up to $25 \%$ in patients with large anterior MI. Our patient initially presented with symptoms of acute limb ischemia. However, on further preoperative evaluation, it was revealed that he had a late presentation of an anterior STEMI complicated by LV thrombus that had embolized to cause limb ischemia. This was managed by coronary revascularization followed by limb revascularization, and hence salvaging the limb without any perioperative complications.

\section{Case Presentation}

A 55-year-old male presented to the emergency department with numbness and pain in the left lower extremity. The pain started suddenly a few hours ago, was very intense, and associated with a sensation of cold extremity. On presentation, the patient had a heart rate of 68 beats per minute, blood pressure of $128 / 77 \mathrm{mmHg}$, respiratory rate of 20 breaths per minute, and a temperature of $98.2^{\circ} \mathrm{F}$. Physical examination revealed the patient to be in distress due to pain and absent dorsalis pedis pulse on the left with pale and cool extremity. Bedside Doppler examination confirmed the absence of dorsalis pedis pulse. A preoperative electrocardiogram (EKG) showed ST-segment elevations in leads V4-V6, II, III, and avF, with reciprocal ST depressions in leads V1-V3, leads I, and avL (Figure 1).

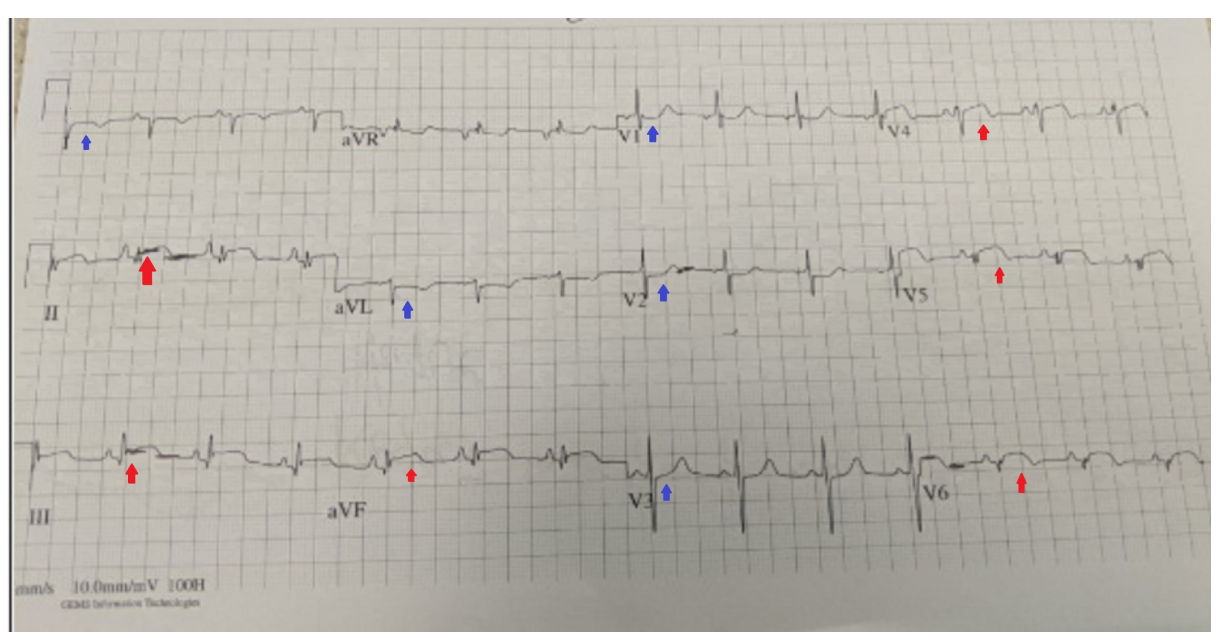




\section{Cureus}

FIGURE 1: Electrocardiogram on presentation showing ST-segment elevations in leads V4-V6, II, III, and avF (red arrows) with reciprocal changes in leads I, avL, and V1-V3 (blue arrows).

On further exploration and a thorough history, the patient endorsed intermittent left arm heaviness and tingling over the last two days, but he neglected it and avoided seeking medical care due to the recent coronavirus-related pandemic. Past history was significant for a six pack-year cigarette smoking (quit 35 years ago), well-controlled human immunodeficiency virus (HIV) infection, and depression. Differential diagnoses included acute limb ischemia due to an embolic phenomenon from aortoiliac thrombus or infective endocarditis or cardiac thrombus with embolization and subsequent critical lower extremity ischemia. Complete blood count and basic metabolic panel were unremarkable. Troponin I was elevated to $22.4 \mathrm{ng} / \mathrm{mL}$ (normal value $<0.07 \mathrm{ng} / \mathrm{mL}$ ). Creatine kinase (CK) was elevated at $328 \mathrm{U} / \mathrm{L}$ (reference range 55$170 \mathrm{U} / \mathrm{L}$ ). Coronavirus testing via nasopharyngeal swab was negative. Computed tomography angiogram (CTA) of the left lower extremity showed acute occlusion of the left common femoral artery with diminutive and poorly visualized downstream arterial vasculature (Figure 2).
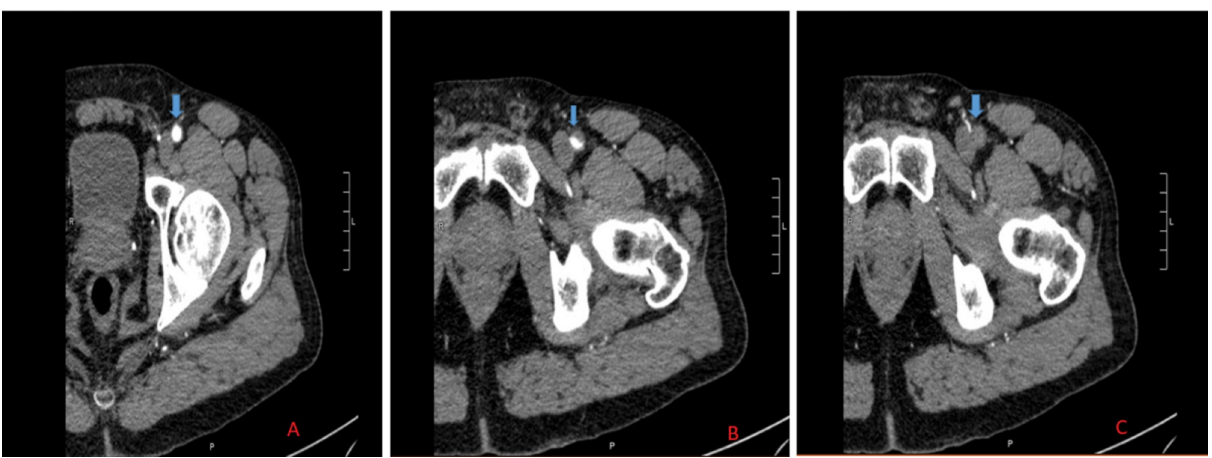

FIGURE 2: Computed tomography angiogram (CTA) of lower extremities showing acute vascular occlusion at the level of left common femoral artery demonstrated by an abrupt cut-off of the contrast (blue arrows) as shown by successive cross-sectional images from panels A-C.

The patient was loaded with aspirin $325 \mathrm{mg}$ and clopidogrel $600 \mathrm{mg}$, and started on intravenous heparin infusion and was emergently taken to the catheterization laboratory. Left heart catheterization (LHC) revealed a subtotal occlusion of the apical left anterior descending artery (LAD) with a mid-systolic bridge. The successful intervention of the distal apical LAD with a 2.0 semi-compliant balloon was performed, hence restoring the flow. Postoperative echocardiogram showed depressed left ventricular ejection fraction (LVEF) of $30 \%$ with severe apical and inferolateral wall hypokinesia and an echodensity $(2.4 \mathrm{~cm} \mathrm{x} 1 \mathrm{~cm})$ in the LV most consistent with a thrombus (Figure 3).
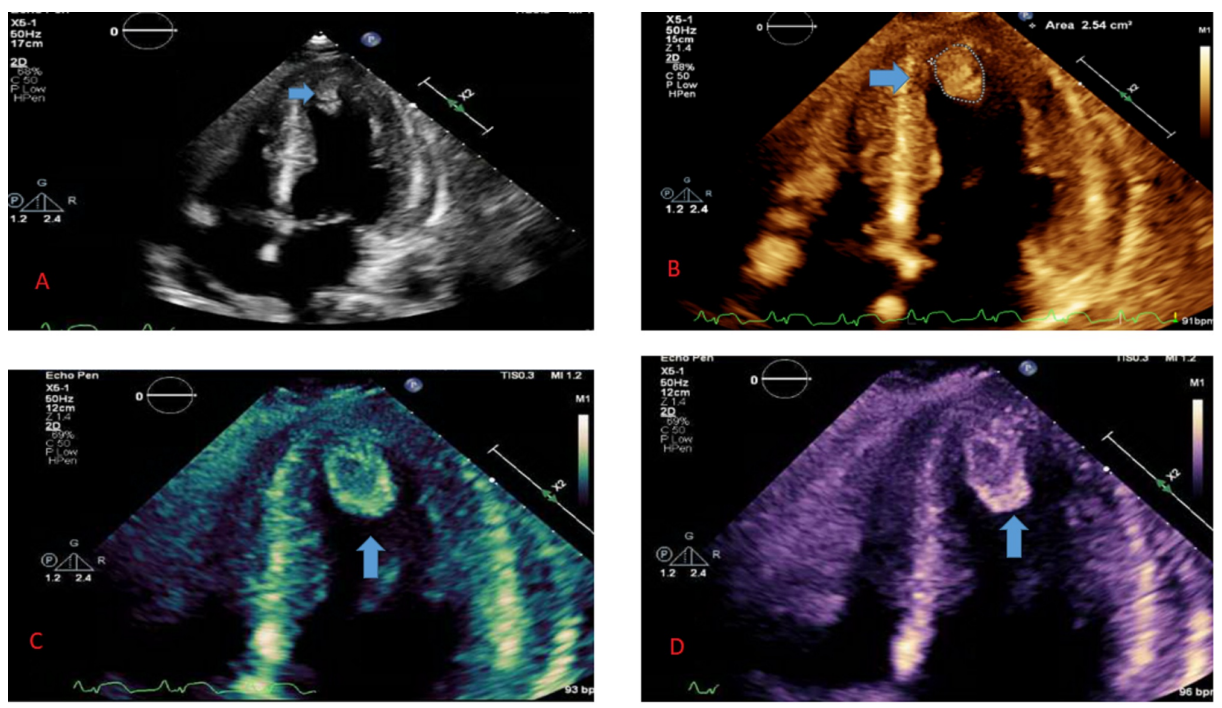
FIGURE 3: Echocardiogram with apical four-chamber view showing thrombus in the left ventricle, which was likely the source of embolus causing acute limb ischemia (panel A showing apical four-chamber view in gray-scale two-dimensional image and panels B-D showing colored images of the apical view for better visualization of the thrombus which is shown by blue arrows).

Immediately post-LHC, the patient was taken to vascular operation room (OR) on the same day where an open thrombectomy of the left common femoral artery thrombus was performed with the restoration of blood flow in the femoral and downstream vasculature demonstrated by palpable distal pulses. Heparin was bridged with warfarin for the LV apical thrombus, and dual antiplatelet therapy with aspirin and clopidogrel was continued. However, on day 3 of triple therapy (warfarin, aspirin, and clopidogrel), the patient developed a left groin hematoma with a drop in hemoglobin. This required close monitoring for hematoma expansion and blood transfusion. Warfarin and aspirin were discontinued, and the patient was cautiously started on apixaban along with clopidogrel (double therapy). An EKG done after the coronary intervention showed persistent V4-V6 leads and T-wave inversions with resolution of ST elevations (Figure 4).

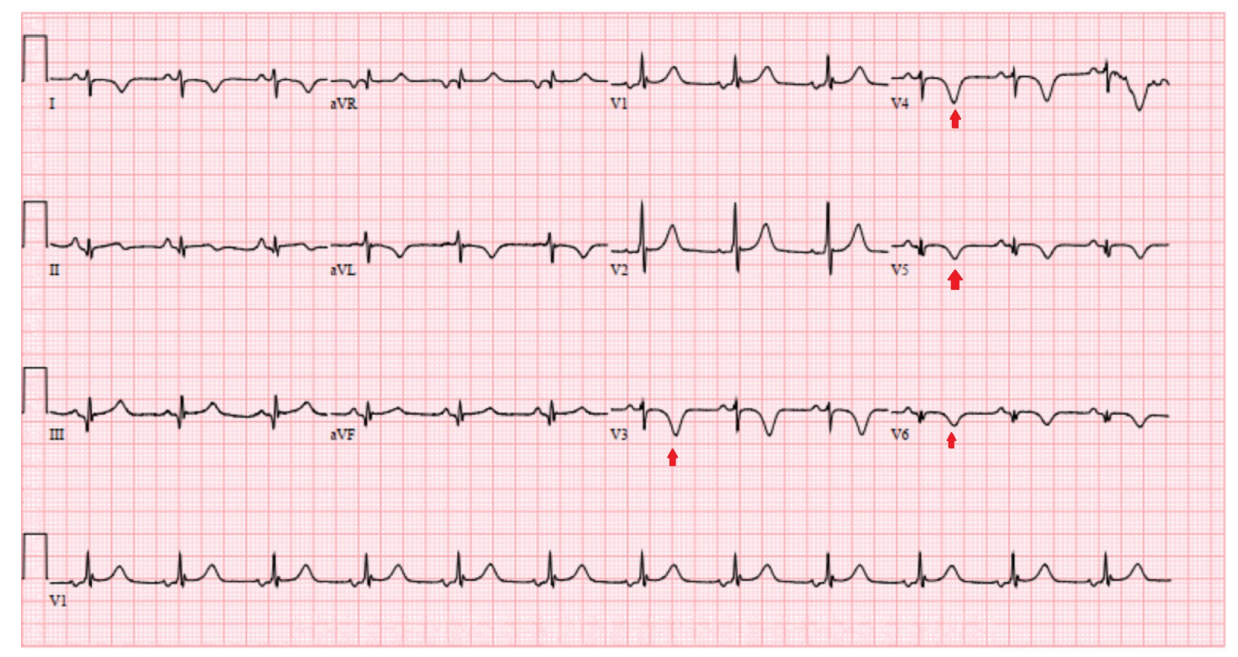

FIGURE 4: Electrocardiogram after revascularization with persistent Twave inversions in the leads V3-V6 with resolution of the ST elevations in these leads (red arrows).

The initial troponin at presentation was $22.4 \mathrm{ng} / \mathrm{mL}$, which started trending down and was $0.16 \mathrm{ng} / \mathrm{mL}$ at day 15 of presentation. The patient was discharged on apixaban and clopidogrel as well as guideline-directed medical treatment with a follow-up surveillance echocardiogram to determine the need for anticoagulation continuation as well as implantable cardioverter-defibrillator placement.

\section{Discussion}

Standard transthoracic echocardiography (TTE) is typically the screening modality of choice for LV thrombus detection and should be performed within 24 hours of admission in those at high risk for apical LV thrombus $[2,3]$. Our patient had subtle symptoms of arm heaviness that he neglected and did not seek medical care of it. His STEMI was brought to clinical attention late and was complicated by ventricular thrombus formation with embolization leading to acute limb ischemia. One likelihood is that patient's severe leg pain has made him oblivious of the subtle chest pressure or pain that he otherwise would have noticed in the absence of the leg pain $[4,5]$. LV apical thrombi have also been associated with systemic thromboembolic events such as stroke leading to significant morbidity and mortality. In rare cases, acute limb ischemia has also been observed requiring emergent surgical management as was seen in our patient [6]. The 2013 American College of Cardiology/American Heart Association (ACC/AHA) STEMI guidelines advise that it is reasonable to add oral anticoagulation $(\mathrm{OAC})$ to dual antiplatelet therapy among patients with STEMI and asymptomatic LV thrombus for three months, targeting a lower international normalized ratio (INR) goal of 2.0-2.5 [7]. The AHA/American Stroke Association 2014 stroke prevention guidelines recommend a similar duration, targeting a higher INR of 2.5 [8]. The European Society of Cardiology 2017 STEMI guidelines advised that once an LV thrombus is diagnosed, OAC should be considered for up to six months, guided by repeated 
echocardiography and with consideration of bleeding risk and need for concomitant antiplatelet therapy [9]. The optimal duration of OAC in these patients is unclear, and decisions regarding the continuation of OAC should be made on a case-by-case basis. There have been no randomized trials to our knowledge that have compared dual and triple therapy in patients with LV thrombosis complicated by thromboembolism and STEMI $[10,11]$. Although data from an observational study have shown an increased risk of systemic thromboembolism with the use of OAC as compared to warfarin, prospective randomized studies are lacking in this area [12]. In our case, due to hemodynamically significant groin hematoma requiring transfusion, the treatment rationale was modified to a limited duration of double therapy (apixaban and clopidogrel) with a follow-up echocardiogram to evaluate the necessity for the continuation of anticoagulation.

\section{Conclusions}

Patients with clinically silent or subtle symptoms of STEMI can present with late complications including LV thrombus and its related embolic complications (strokes/transient ischemic attacks, peripheral arterial disease, or mesenteric ischemia), particularly if the presentation is delayed. Physicians should be vigilant in their preoperative examination and evaluation to evaluate the cause of acute limb ischemia in a previously otherwise healthy patient with no history of peripheral arterial disease and otherwise no thromboembolic predisposition. An EKG evidence of STEMI should raise the suspicion of LV thrombus, which is one of the complications of large anterior MI.

\section{Additional Information}

\section{Disclosures}

Human subjects: Consent was obtained by all participants in this study. Conflicts of interest: In compliance with the ICMJE uniform disclosure form, all authors declare the following: Payment/services info: All authors have declared that no financial support was received from any organization for the submitted work. Financial relationships: All authors have declared that they have no financial relationships at present or within the previous three years with any organizations that might have an interest in the submitted work. Other relationships: All authors have declared that there are no other relationships or activities that could appear to have influenced the submitted work.

\section{References}

1. McCarthy CP, Vaduganathan M, McCarthy KJ, Januzzi JL Jr, Bhatt DL, McEvoy JW: Left ventricular thrombus after acute myocardial infarction: screening, prevention, and treatment. JAMA Cardiol. 2018, 3:642-649. 10.1001/jamacardio.2018.1086

2. Agarwal KK, Douedi S, Alshami A, DeJene B, Kayser RG: Peripheral embolization of left ventricular thrombus leading to acute bilateral critical limb ischemia: a rare phenomenon. Cardiol Res. 2020, 11:134137. $10.14740 / \mathrm{cr} 1030$

3. McCarthy CP, Murphy S, Venkateswaran RV, et al.: Left ventricular thrombus: contemporary etiologies, treatment strategies, and outcomes. J Am Coll Cardiol. 2019, 73:2007-2009. 10.1016/j.jacc.2019.01.031

4. Aziz F, Doddi S, Kallu S, Penupolu S, Alok A: Myocardial infarction (MI) presenting as acute limb: an extremely rare presentation of MI. J Thorac Dis. 2010, 2:57-59.

5. Miyake K, Kikuchi S, Kitani Y, et al.: Successful simultaneous revascularization for acute limb ischemia and concomitant acute myocardial infarction. Ann Vasc Dis. 2018, 11:361-364. 10.3400/avd.cr.18-00059

6. Driesman A, Hyder O, Lang C, Stockwell P, Poppas A, Abbott JD: Incidence and predictors of left ventricular thrombus after primary percutaneous coronary intervention for anterior ST-segment elevation myocardial infarction. Clin Cardiol. 2015, 38:590-597. 10.1002/clc.22450

7. O'Gara PT, Kushner FG, Ascheim DD, et al.: 2013 ACCF/AHA guideline for the management of ST-elevation myocardial infarction: a report of the American College of Cardiology Foundation/American Heart Association Task Force on Practice Guidelines. J Am Coll Cardiol. 2013, 61:78-140. 10.1016/j.jacc.2012.11.019

8. Kernan WN, Ovbiagele B, Black HR, et al.: Guidelines for the prevention of stroke in patients with stroke and transient ischemic attack: a guideline for healthcare professionals from the American Heart Association/American Stroke Association. Stroke. 2014, 45:2160-2236. 10.1161/STR.0000000000000024

9. Ibanez B, James S, Agewall S, et al.: 2017 ESC Guidelines for the management of acute myocardial infarction in patients presenting with ST-segment elevation: the Task Force for the management of acute myocardial infarction in patients presenting with ST-segment elevation of the European Society of Cardiology (ESC). Eur Heart J. 2018, 39:119-177. 10.1093/eurheartj/ehx393

10. Shokr M, Ahmed A, Abubakar H, Sayedahmad Z, Rashed A, Afonso L, Cardozo S: Use of direct oral anticoagulants in the treatment of left ventricular thrombi: a tertiary center experience and review of the literature. Clin Case Rep. 2019, 7:135-142. 10.1002/ccr3.1917

11. Cochran JM, Jia X, Hamzeh I, Birnbaum Y: Direct oral anticoagulant use for left ventricular thrombus: a single center experience. Circulation. 2018, 138:A16411.

12. Robinson AA, Trankle CR, Eubanks G, et al.: Off-label use of direct oral anticoagulants compared with warfarin for left ventricular thrombi. JAMA Cardiol. 2020, 5:685-692. 10.1001/jamacardio.2020.0652 